\title{
Underwater telemetry as a tool in aquaculture research and development
}

\section{B. A. HOLAND $\dagger$}

Keywords: Fish telemetry, underwater acoustics, biology, behavioural sciences, tracking systems.

Small acoustical transmitters may be used to obtain data from free swimming fish. The main principles behind this technique are described together with some of the equipment developed. Some examples are briefly discussed where heart beats, depth and temperature are measured to describe fish behaviour. Automatic fish tracking equipment that has been used to track fish within an enclosed area is also described.

\section{Introduction}

Since 1973 a project called 'Fish Telemetry' has been in progress at SINTEF div. Automatic Control supported mainly by Norges Fiskeriforskningsråd (Norwegian Fisheries Research Council), NFFR. The main goals for the project have been to develop instrumentation in order to obtain data from free swimming fish, and to assist fisheries' researchers both in solving their specific instrumentation requirements, and in applying the equipment in practical experiments either in the field or in the laboratory. These activities have been annually reported to NFFR, and Mohus and Holand (1983) is a summary of most of these reports.

\section{Fish telemetry}

\subsection{The method}

Fish telemetry may generally be described by Fig. 1. A fish is equipped with a transmitter which relays information about one or more parameters through the water. The information is received and processed with a varying degree of complexity by the receiving equipment.

Parameters that are most frequently measured in our experiments are

Position

Depth

Temperature (surrounding or internal)

Heart rate

Optional parameters are

Activity (tail beats)

Breathing (gill movements)

Smell (reactions from the olfactory bulb)

Light

Received 15 January 1987.

This paper was presented at the IFAC Symposium on Automation and Dataprocessing in Aquaculture, Trondheim, Norway, 18-20 August 1986.

This paper is reprinted with the permission of IFAC.

$\uparrow$ SINTEF div. Automatic Control, NTH, Trondheim, Norway. 


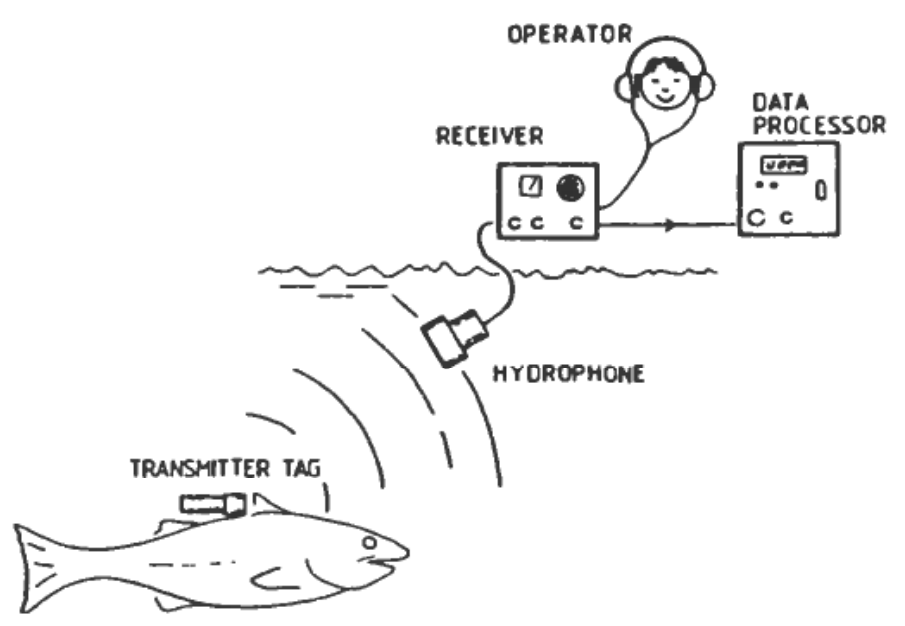

Figure 1. Fish telemetry.

\subsection{The transmitter}

Although radio transmitters may be used in fresh water to relay information, acoustical transmitters are the only choice in sea water. In principle any sound frequency can be used, but in our transmitters the major frequency range is between $100 \mathrm{kHz}$ and $150 \mathrm{kHz}$. The higher absorption losses at such high frequencies are generally compensated for both by lower noise levels (noise induced by man and the weather), and the fact that absorption losses are less significant at these frequencies than the spreading losses for transmission over distances less than $1 \mathrm{~km}$.

For our general transmitters, detection ranges from 100-1500 m are common. In traditional aquaculture however, range is not a problem as the fish are kept in areas with dimensions that are small compared with the ranges mentioned above.

As the transmitters are battery powered, when possible the output signal is pulsed on and off to conserve energy. The operating time will vary based on parameters like battery size, output power, on/off ratio, etc., and may range from hours to a year.

Information in addition to position is generally relayed by letting the physical parameter(s) measured modulate the pulse repetition rate, and/or the output carrier frequency. It is thereby possible to measure three individual parameters in a fairly simple transmitter by letting one parameter (e.g. the temperature) modulate the pulse repetition rate, while the second (e.g. depth) is modulating the carrier frequency. In addition, the output signals themselves can be used for positioning.

Transmitters measuring physiological variables like ECG or EMG, often have a continuous output signal which is frequency modulated by the measured variable. Such transmitters therefore, due to continuous battery consumption, will have reduced operating life compared to pulsed transmitters. For fish (salmon) weighing approximately $4 \mathrm{~kg}$, we have made heart beat transmitters lasting three months.

Fig. 2 gives a list of some general well proven transmitters.

\subsection{Receiving equipment}

Receiving equipment generally consists of a hydrophone (equivalent to microphone in air) and a receiver that is basically an electronic amplifier that selects the 
proven transmitters is given.

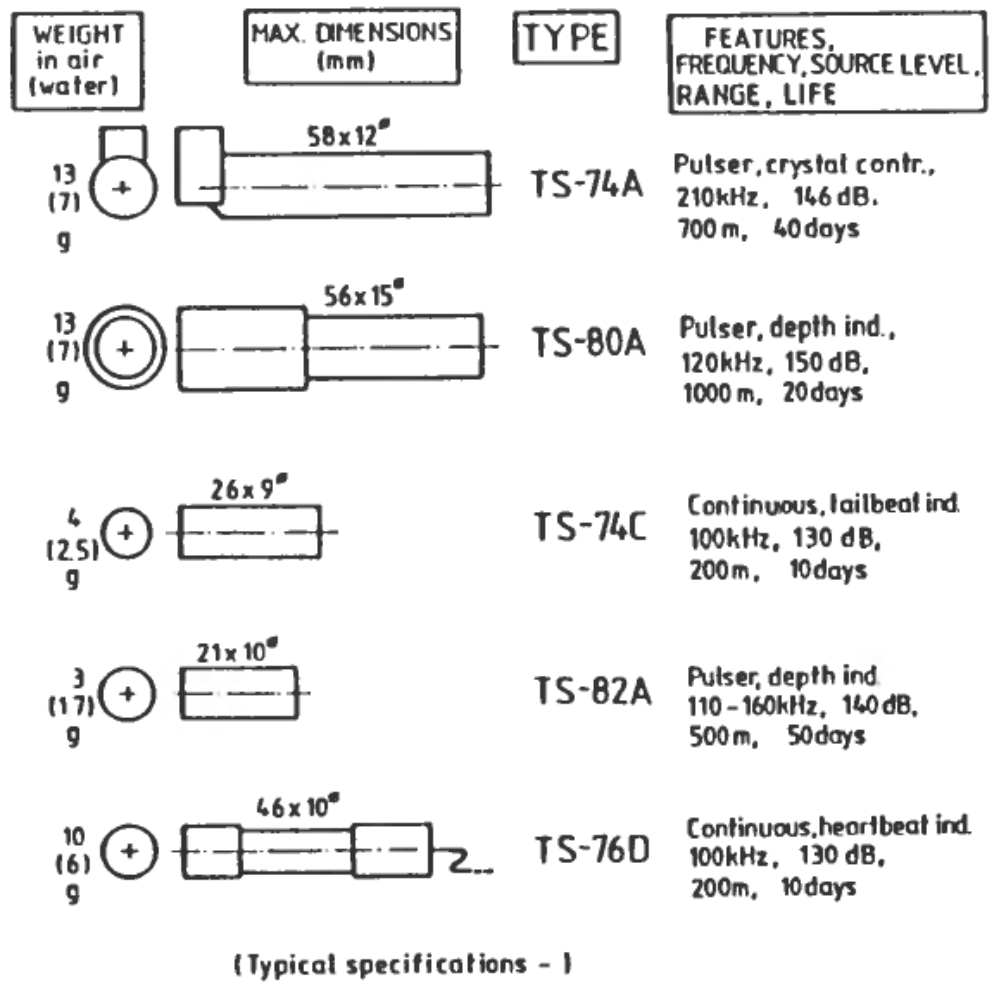

Figure 2. Some well proven transmitters.

desired signals received by the hydrophone, and presents an audio output signal to the operator. Also an electronic output signal to processing equipment is often provided. Processing equipment will vary with the parameters measured, and the nature of the experiment. Some examples will be given later in this paper.

\section{Some experiments}

Some experiments which have been made by the author will illustrate how fish telemetry may be used in aquaculture research and development.

\subsection{Heart rate monitoring}

The heart beats (ECG) of a fish may be measured by inserting one electrode close to the heart of the fish, and another placed anywhere on the fish acting as the reference.

It is well known that the heart rate will vary depending on both physiological and psychological parameters (e.g. physical activity and stress), and heart rate has proven to be a very useful parameter when fish behaviour is studied. It is often possible to see almost dramatic changes in heart rate without any visible signs of changed physical behaviour. This can be used to study detection levels of parameters like smell, sound, electrical fields, etc. 


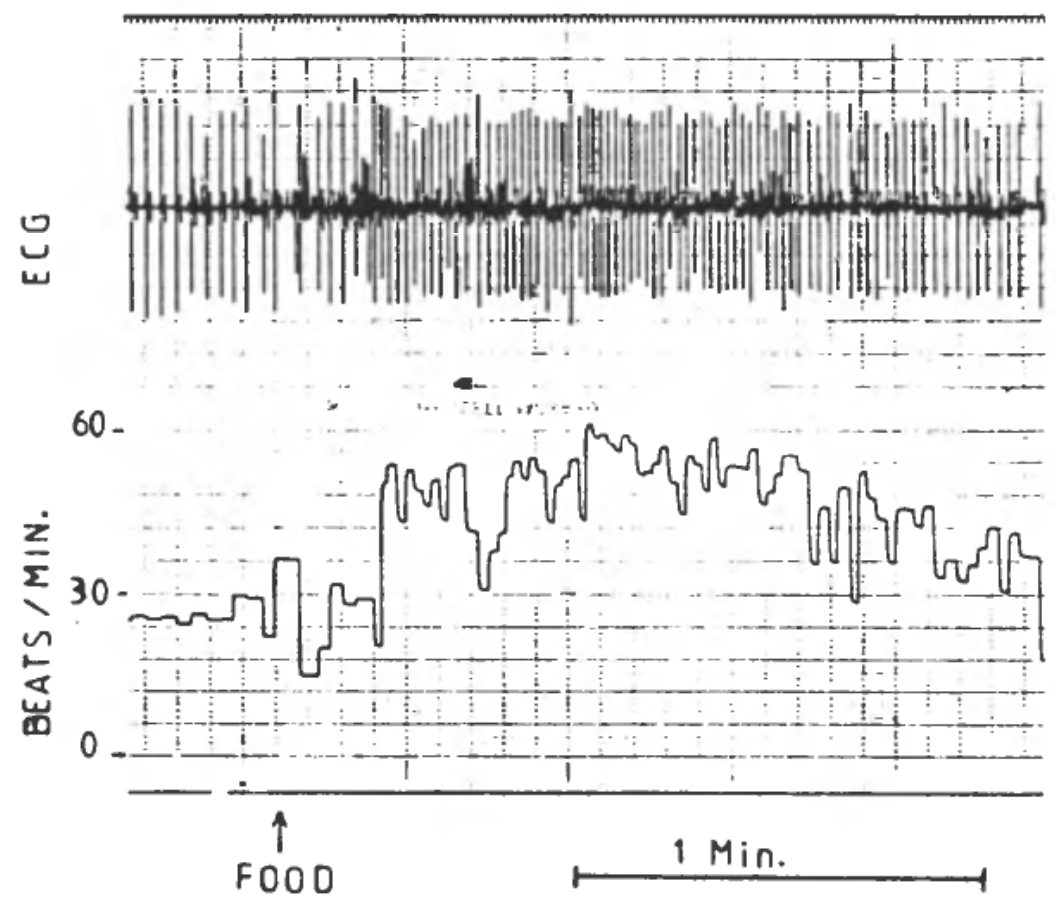

Figure 3. Heart rate of a saithe when feeding.

At other times, certain heart rate patterns seem to be related to defined physical behaviour. One example of that was demonstrated in a number of experiments done with feeding saithe (Gadus pollachius virens). The fish were tagged with heart beat transmitters, and released, some in a pen (together with a large number of other fish), others were swimming freely in the sea. The fish were trained to associate a certain acoustic signal with food before they were tagged. In Fig. 3 a typical heart rate reaction to feeding is shown.

The upper channel of the recording shows the actual heart beats (ECG), while the lower one shows the equivalent beat-to-beat frequency (beats/min). Typically, when the fish detected the food, one or more heart beats were skipped. After that the 'struggle' for food started, and during that period the heart rate was approximately doubled. Then, when the food was eaten, the heart rate faded back to normal again over a period of 2-3 min. This reaction was found to be typical for all the fish tagged, not only those kept in the pen, but also for free swimming saithe. Free swimming fish showed no similar reaction to other (natural) stimuli, and not even continuous swimming (at least $1 \mathrm{~km}$ in $1 \mathrm{~h}$ ) increased the heart rate significantly compared with the reaction shown in Fig. 3.

The experiment described above was performed in order to find a way to detect feeding activity of free swimming fish, but the findings have recently become interesting in another application. In traditional aquaculture fish are generally kept in high concentrations within pens. High concentrations of living organisms are, among other things, known to create stress among the individuals, and stress is known to reduce the natural immune apparatus against various infections. Consequently, stress is considered to be a serious problem within fish farming (aquaculture). The rapid increase in heart rate shown in Fig. 3 is possibly caused by 
stress hormones (adrenalin). A continuous high heart rate (e.g. caused by continuous feeding) will increase the amount of stress hormones (e.g. cortisol) in the organism which may result in the problems mentioned above.

If a reliable correlation between heart rate and the amount of stress hormones in the fish can be demonstrated, heart beat transmitters on a small number of fish in a pen could be used to detect stress, and if possible, the situation could be corrected in due time before the situation becomes critical.

\subsection{Other parameters}

In traditional aquaculture, the fish's choice of depth is limited by the depth of the pen. How deep a pen should be may vary. Thermal parameters will in some areas be a problem for the fish. During summer, the temperature may be too high in the upper layer to supply enough oxygen, and cold may be a problem during extreme frost in the winter, or during spring in areas where the temperature in the water is affected by massive snow melting in the mountains.

To establish knowledge about the problems described above, fish in an actual area could be equipped with a combined depth/temperature transmitter. If the fish are given a choice regarding any depth preference during the different seasons, data from such a transmitter could facilitate a correct depth design of pens used in that area at a given time.

In a more advanced aquaculture situation, where fish are allowed to move freely within a selected area instead of being limited by the pen, depth recordings may be useful to establish the design of feeding equipment. The question may be at what depth is the fish willing to catch food, either sinking from the surface, or floating up from some depth. In Fig. 4 a depth recording of feeding saithe is shown. Here it is shown that the fish pursue the sinking food down to approximately $10 \mathrm{~m}$.

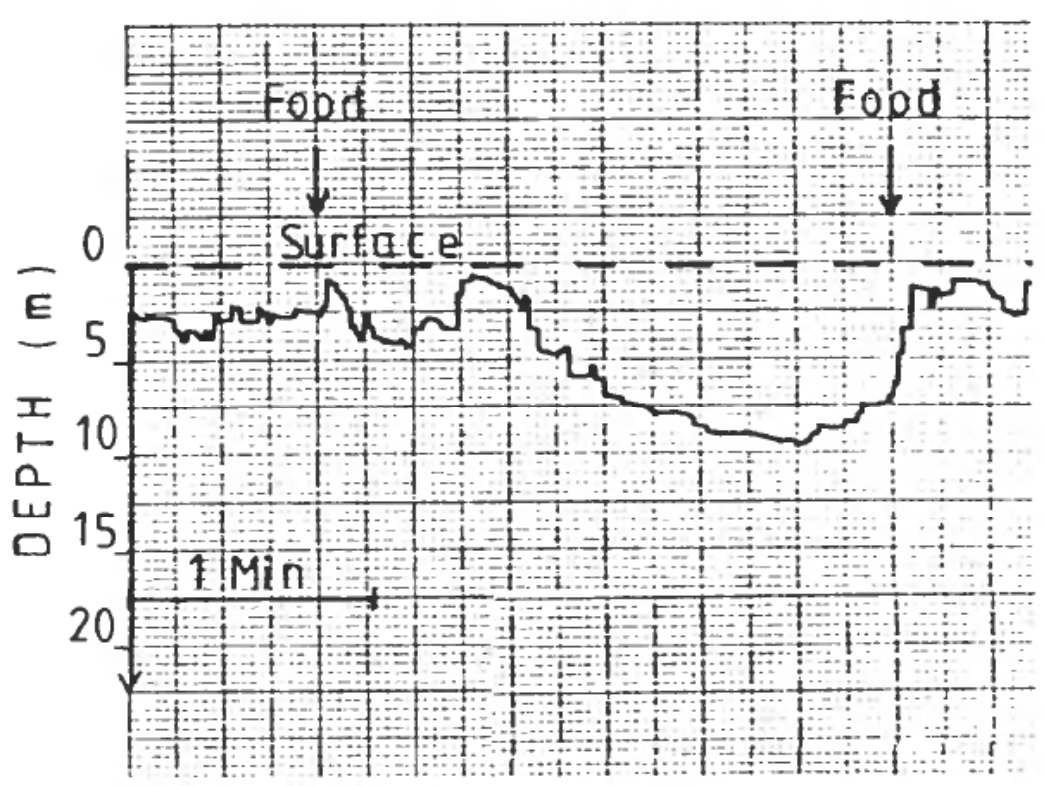

Figure 4. An automatic depth recording from a feeding saithe. 

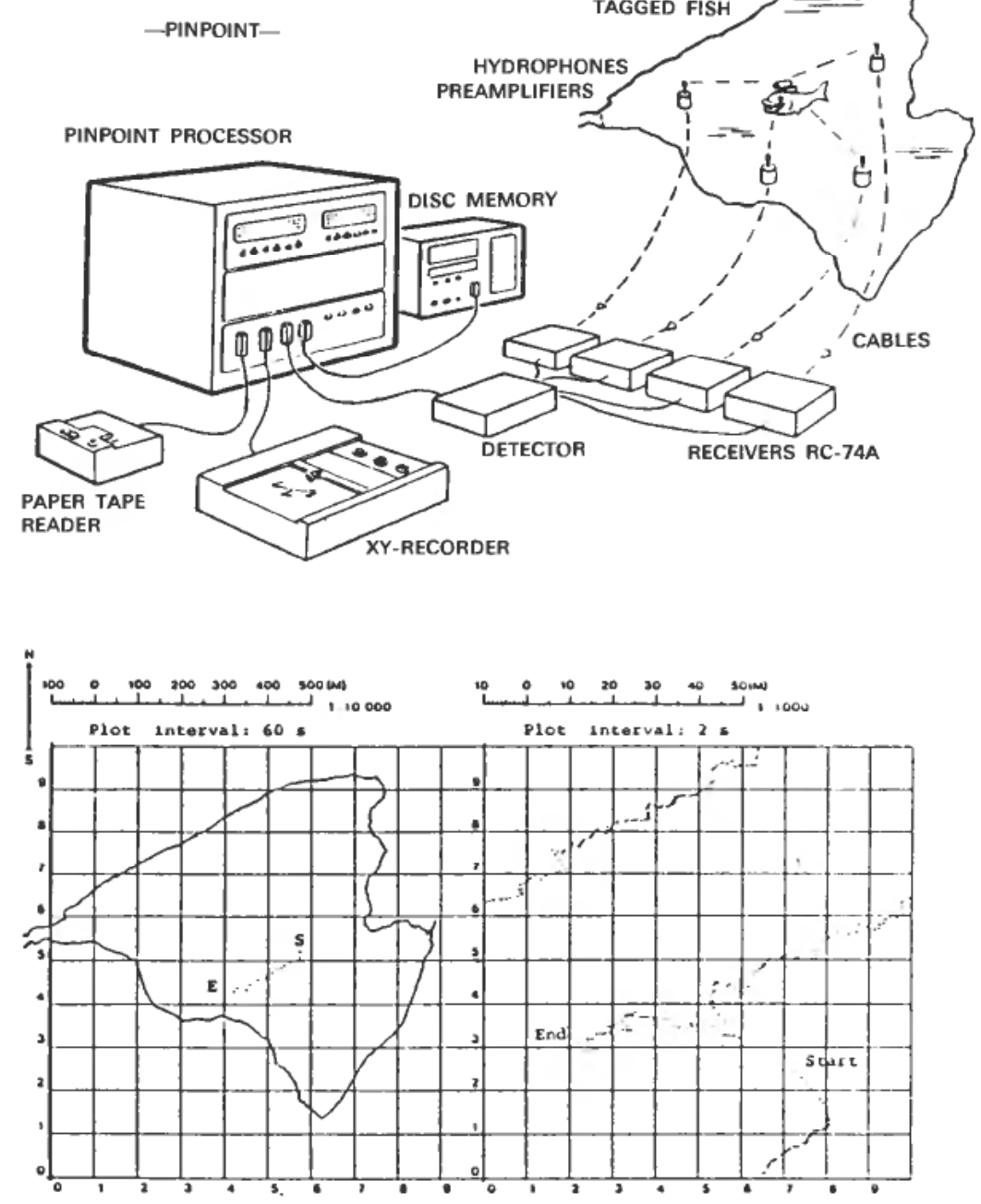

Figure 5. The PINPOINT system.

\subsection{Automatic fish tracking}

The principles of the automatic fish tracking system PINPOINT are shown in Fig. 5. A pulse from the transmitter is received by up to four hydrophones, and based on the differences in time of arrival of the pulse at the different hydrophones, the PINPOINT processor calculates the actual position of the fish. The position is plotted on a XY recorder. One such plot is shown in Fig. 5. An over-all-view is presented in the left hand main square, where each sub-square is $100 \times 100 \mathrm{~m}$. A more detailed tracking is presented in the right hand main square, where each subsquare is $10 \times 10 \mathrm{~m}$.

A continuous automated tracking system is very useful when detailed fish behaviour related to different parameters is to be studied. Also in the advanced aquaculture situation mentioned above, such equipment may prove useful answering such questions as 
How do the fish react regarding food?

How often do the fish feed?

What about the distribution of feeding stations?

What about the reaction to thermal abnormalities?

\section{ACKNOWLEDGMENTS}

The author wishes to thank: the Norwegian Fisheries Research Council (NFFR) for funding the fish telemetry project since 1973; Prof. J. G. Balchen who initiated the project; M.Sc. I. Mohus for technical cooperation since 1973, and for providing figures for this paper; Dr. J. Kanwisher for close cooperation since 1973.

\section{REFERENCE}

MoHUS, I., and Holand, B. (1983). Fish Telemetry Manual. SINTEF report STF48 A83040 for NFFR, Dec. 1983. 